\title{
街头抢劫者前犯罪经历对其后作案地选择的影响
}

\author{
龙冬平 ${ }^{1}$, 柳 林 $^{1,2^{*}}$, 陈建国 ${ }^{1}$, 肖露子 ${ }^{1}$, 宋广文 ${ }^{1}$, 徐 冲 $^{1}$ \\ (1. 广州大学地理科学学院, 公共安全地理信息分析中心, 广州 510275 ; \\ 2. 辛辛那提大学地理系, 美国 辛辛那提 $\mathrm{OH}$ 45221-0131)
}

\begin{abstract}
摘 要: 作案地选择是犯罪地理学的研究主题。已有的重复作案地选择的研究表明,犯罪者“前案件”作案地选择对 他们“后案件”作案地选择具有影响, 但以往研究关注的是先前的犯罪时间和地点对其后续作案地选择的影响,仍 未检验犯罪者在 “前案件” 中犯罪经历的具体作用。因此, 论文以中国东南沿海 ZG 市为例, 利用街头抢劫者的抓 捕数据和混合 Logit模型, 聚焦探析街头抢劫者先前的个体犯罪经历对他们随后的作案地选择的影响。研究发现: 街头抢劫者在“前案件”中的犯罪间隔、犯罪出行和当场被捕等个体犯罪经历对其“后案件”作案地选择具有强烈的 影响, 即“前后案件”的犯罪间隔越临近、“前案件”犯罪出行距离越短, 以及“前案件”未当场被捕, 则大大增加了街 头抢劫者返回到先前抢劫区域再次犯罪的可能性。并通过警察访谈和结合理论分析, 发现“前案件”未当场被捕是 由犯罪者当场被捕的恐惧感、警察特殊的干预方式, 以及社会凝聚力和犯罪防控的相互作用而形成。研究结论可 为警务部门的“事前防控”与 “主动处置”提供一定的参考。
\end{abstract}

关 键 词: 街头抢劫者; 犯罪经历; 作案地选择; 混合 Logit模型; 犯罪地理学

理解犯罪者如何选择作案地是犯罪地理学的 经典问题 ${ }^{[1]}$ 。关于街头抢劫者作案地选择的现有研 究, 主要集中在以下 2 个方面:一是从综合视角分析 街头抢劫者作案地选择的空间模式与影响因素 ${ }^{[-6]}$; 二是从对比视角分析街头抢劫者作案地选择的时 间差异 ${ }^{[7-9]}$ 。总的来看, 这些研究不仅有利于丰富街 头抢劫者作案地选择的研究视角, 也有助于明确其 时空规律与形成机制, 因此, 具有重要的理论与实 践意义。然而, 在已有研究中, 仍缺乏重复作案地 选择背景下先前犯罪经历作用或影响的分析。例 如, Lammers 等 ${ }^{[10]}$ 认为过去的研究忽视了犯罪者当
前作案地选择受先前犯罪活动或犯罪经历的影 响。类似地, Bernasco 等 ${ }^{[1]}$ 在入室盗窃的研究中也 强调了这个问题, 即大多数人室盗窃者作案地选择 的研究, 尚未探讨一个人室盗窃者先前的犯罪经历 可能会影响其后续在何处犯罪。与此同时, 虽然在 重复受害的研究中已发现了这一现象, 即初始和后 续街头抢劫都是由相同犯罪者实施的 ${ }^{[12-13]}$, 但大多 数研究将街头抢劫看作是一个孤立的事件, 即忽视 了所涉及的街头抢劫者过去的犯罪经历或犯罪活 动的影响 ${ }^{[14]}$ 。这有可能是一个非常严重的疏忽。

犯罪者为什么会重返到先前的犯罪区域作

收稿日期: 2019-05-06; 修订日期: 2019-09-24。

基金项目: 国家自然科学基金青年科学基金项目(41901172,41901177,41601138); 广东省自然科学基金研究团队项目(2014A030312010); 国家自然科学基金重点项目(41531178); 国家重点研发计划项目(2018YFB0505500,2018YFB0505503); 广州市科学研究计划重点项目(201804020016)。[Foundation: National Natural Science Foundation of China, No. 41901172, 41901177 and 41601138; Research Team Program of Natural Science Foundation of Guangdong Province, China, No. 2014A030312010; Key Program of the National Natural Science Foundation of China, No. 41531178; National Key R\&D Program of China, No. 2018YFB0505500 and 2018YFB0505503; Guangzhou Science and Technology Program Key Projects, No. 201804020016. ]

第一作者简介: 龙冬平(1985-), 男, 湖南邵阳人, 博士, 讲师, 主要从事公共安全与犯罪地理研究。E-mail: longdp@gzhu.edu.cn *通信作者简介: 柳林(1965-), 男, 湖南湘潭人,博士, 教授, 中国地理学会会员 $(\mathrm{S} 110007983 \mathrm{M})$, 主要从事人文地理信息科学、 犯罪时空分析与模拟研究。E-mail: lin.liu@uc.edu

引用格式: 龙冬平, 柳林, 陈建国, 等. 街头抢劫者前犯罪经历对其后作案地选择的影响 [J]. 地理科学进展, 2020, 39(5): 815-828. [Long Dongping, Liu Lin, Chen Jianguo, et al. Impact of prior crime experiences of street robbers on subsequent crime location choices. Progress in Geography, 2020, 39(5): 815-828. ] DOI: 10.18306/dlkxjz.2020.05.010 
案? 最近一些学者对这一问题进行了讨论, 并提出 一些观点。例如, Bernasco 等 ${ }^{[3]}$ 认为在犯罪者的感 知空间中, “前案件” 区域可能是一个重要的犯罪场 所, 它或许决定了他们“后案件”作案地选择。John$\operatorname{son}^{[15]}$ 认为犯罪者很可能去评估“前案件” 区域的安 保措施、高收益区和逃离路径等, 并有可能在未来 的犯罪中充分利用这些信息。Townsley 等 ${ }^{\left[{ }^{[16]}\right.}$ 认为 犯罪者可从 “前案件” 中学习或根据以前的犯罪经 历调整他们的偏好以获取最大的收益。总的来看, 这些研究虽提出了“前案件”犯罪经历对“后案件” 作案地选择有影响的观点, 但尚未对其进行检验。

近年来, 在重复犯罪实证研究方面, 少许学者 利用警方提供的抓捕数据, 分析了犯罪者“前案件” 的时间和地点对他们 “后案件” 作案地选择的影 响。例如, Lammers 等 ${ }^{\left[{ }^{[0]}\right.}$ 以荷兰海牙地区为例, 发现 犯罪者趋向返回先前的犯罪区域, 特别是时空上越 临近的“前后案件”, 以及“前后案件”类型相同的犯 罪。Bernasco 等 ${ }^{[1]}$ 在英国西米德兰兹郡的研究中也 得到类似的结论, 即“后人室盗窃” 作案地很可能发 生在先前的人室盗窃区域, 特别时间上越接近先前 的人室盗窃, 以及越靠近先前人室盗窃作案地的区 域也很可能被选择。总的来看, 基于时间和地点的 研究, 虽在一定程度上能够代表一个犯罪者先前的 一些犯罪经历对其后续作案地选择的影响, 但仍尚 未验证犯罪者在“前案件” 区域哪些犯罪经历的具 体作用。

鉴于此, 本文的研究目的是进一步探索重复作 案地选择中的个体犯罪经历问题, 并在 3 个关键方 面与 Lammers 等 ${ }^{[10]}$ 和 Bernasco 等 ${ }^{[1]}$ 的研究不同。首 先, 本文在继承已有研究的基础上, 分析街头抢劫 者在“前案件”中的犯罪间隔、犯罪出行和当场被捕 等个体犯罪经历是否对其 “后案件” 作案地选择具 有影响。其次, 犯罪类型不同。Lammers等的研究 包含所有犯罪类型, Bernasco 等研究的是人室盗 窃, 而本文关注的是街头抢劫。最后, 研究区域不 同。Lammers 等和 Bernasco 等研究的是西方国家, 而本文研究的是中国。总之, 本文对街头抢劫者重 复作案地选择制定了明确的假设, 并将理论和实证 研究下的犯罪诱因融合到街头抢劫者作案地选择 的一般模型中, 以及结合街头抢劫者的抓捕数据, 采用混合 Logit模型对本文提出的 3 个假设进行严 格的检验。

\section{1 理论基础、概念框架与研究假设}

为更好地理解街头抢劫者作案地选择, 首先有 必要讨论犯罪模式理论、理性选择理论和社会解组 理论。因此, 本文结合上述理论对相关文献进行梳 理, 并提出本文的概念框架与研究假设。

\section{1 理论基础}

\subsection{1 犯罪模式理论}

犯罪模式理论解释了犯罪者为什么会在同一 个地方多次犯罪 ${ }^{[10]}$ 。首先,一个区域可能拥有若干 个学校、大型购物中心和公共交通站 ${ }^{[3,17-19]}$ 。这些场 所被视为“犯罪产生地” ${ }^{[20]}$, 它们作为公众容易进人 的场所, 因大量人群的存在可能成为犯罪热点, 这 对街头抢劫者来说, 将会创造更多的犯罪机会 ${ }^{[21]}$ 。 其次,一个区域也可能拥有大量的日常活动场所, 如酒吧与俱乐部、网吧、杂货店、宾馆和饭店、银行 和 ATMs 等场所 ${ }^{[3,7-8,14,21-22]}$ 。这些场所被看作是 “犯罪 吸引地” ${ }^{[20]}$, 虽不一定能同时汇聚大量的人群, 但这 些节点的现金交易频繁,对街头抢劫者来说,将是 一个理想的“狩猎场”。例如, Jean ${ }^{[23]}$ 在芝加哥的研究 中发现,抢劫者被吸引到以现金交易为主的小商场。 因此, 这些日常活动节点就非常适合有动机的街头 抢劫者, 去寻找有吸引的或缺乏保护的目标。最 后, 犯罪者的感知空间是有限的。街头抢劫者对邻 近他们居住地及周围的日常活动节点有熟悉的认 知 ${ }^{[3]}$ 。犯罪者通常不会远离这个感知空间 ${ }^{[24]}$, 因为远 离是有风险的。此外, Bernasco 等 ${ }^{[2-3]}$ 认为街头抢劫 者的感知空间决定了他们对收益、成本和风险的感 知, 这说明了犯罪者的感知空间对其作案地选择起 着关键的作用。因此, 街头抢劫者通常会到离现居 住地不远的地方去寻找合适的目标, 这不仅有利于 他们更容易找到高收益的地方，而且有利于他们抢 劫后迅速逃离现场, 从而也就降低了被捕的风险。 总之, 犯罪模式理论下的犯罪诱因, 有助于解释街 头抢劫者为什么会在同一个区域再次犯罪。

\subsection{2 理性选择理论}

理性选择理论认为犯罪者的决策是理性的,但 因个体特征、资金需求、犯罪机会和社会因素等方 面不同,这种理性是有限的 ${ }^{[25]}$ 。Clarke等 ${ }^{[26]}$ 和Felson 等 ${ }^{[27]}$ 进一步指出犯罪者是基于收益、风险和成本的 感知对是否犯罪作出决策。Morrison 等 ${ }^{[28]}$ 发现大多 数犯罪者能够精确评估抢劫潜在的收益和选择合 适的目标,并指出犯罪者会采取降低被抓风险的策 
略,如选择在一天最安静的时间进行抢劫 ${ }^{[28]}$ 。同时, 理性选择理论认为, 如果提供一个无风险的犯罪机 会,任何人都会犯罪。例如通常一个不会犯罪的人， 在收益高又不会被抓的情况下也可能会犯罪 ${ }^{[27]}$ 。 此外, 理性选择理论与传统犯罪学理论认为犯罪行 为不可避免的观点不同, 当被捕风险高或犯罪收益 小的时候,一个人可能不会犯罪 ${ }^{[26-27]}$ 。

理性选择理论认为犯罪者从他们先前犯罪中 吸取经验与教训, 以实现后续犯罪收益更大或被捕 风险更低 ${ }^{[29]}$ 。例如, Townsley 等 ${ }^{[16]}$ 认为犯罪者可从 “前案件”中学习或根据以前的犯罪经历调整他们 的偏好以获取最大的收益等。加之, 犯罪者偏向返 回先前成功的区域,因在这些区域他们能找到有钱 的地方或能快速地获取现金 ${ }^{[3,21,30]}$ 。因此,理性的街 头抢劫者可能会对他们 “前案件” 中的犯罪经历加 以分析, 如先前抢劫区域, 是否有群众追赶或警察 围捕, 是否会当场被捕或能轻松逃离, 以及犯罪出 行距离是否较远等? 通过这一系列的分析, 从而决 定他们是否返回到“前案件” 区域去物色目标及实 施犯罪。

\subsection{3 社会解组理论}

社会解组理论, 亦称社会失序理论, 该理论关 注邻里的社会结构如何抵制或促进犯罪活动 ${ }^{[31-32]}$, 它
的核心概念是社会凝聚力 ${ }^{[10,33]}$, 如它存在将有利于 居民之间形成集体效能去阻止犯罪。现有研究认 为, 社会凝聚力很可能出现在这些社区, 即人口稳 定的社区, 居民能形成良好的社会关系 ${ }^{[34-36]}$, 以及阶 层均质的社区，居民能形成共同的目标与价值 ${ }^{\left[{ }^{[7-38]}\right.}$ 。

社会解组理论从 3 个方面解释了犯罪发生的机 理。第一,邻里间良好的社会关系能够形成集体效 能去减少犯罪机会 ${ }^{[34]}$,如居民可以对生活在附近的 人发挥非正式控制, 以减少他们参与犯罪的可能 性 ${ }^{[39]}$ 。第二,社会经济异质性较强的社区,由于居 民之间缺乏共同价值,他们较难联合起来去抵制犯 罪活动 ${ }^{[40]}$ 。例如, 柳林等 ${ }^{[4]]}$ 发现中国 ZG 市的城中 村、老城区是犯罪分子的主要“狩猎场”, 主要原因 是这些区域的人群流动性较大、外来人口比重较高 以及社会经济异质性较高。第三,犯罪者对社会凝 聚力的感知, 可能影响他们决定在何处犯罪。例 如, Bernasco 等 ${ }^{[42]}$ 认为社会凝聚力可以作为一种阻 抗形式,抑制犯罪者在社会凝聚力高的社区犯罪。

\section{2 概念框架}

根据以上的理论和文献, 提出如图 1 所示的概 念框架。这一概念框架实质是重复犯罪的视角, 即 分析街头抢劫者“前案件”的犯罪经历是否对其“后 案件”作案地选择具有影响。它考虑了 3 组变量因

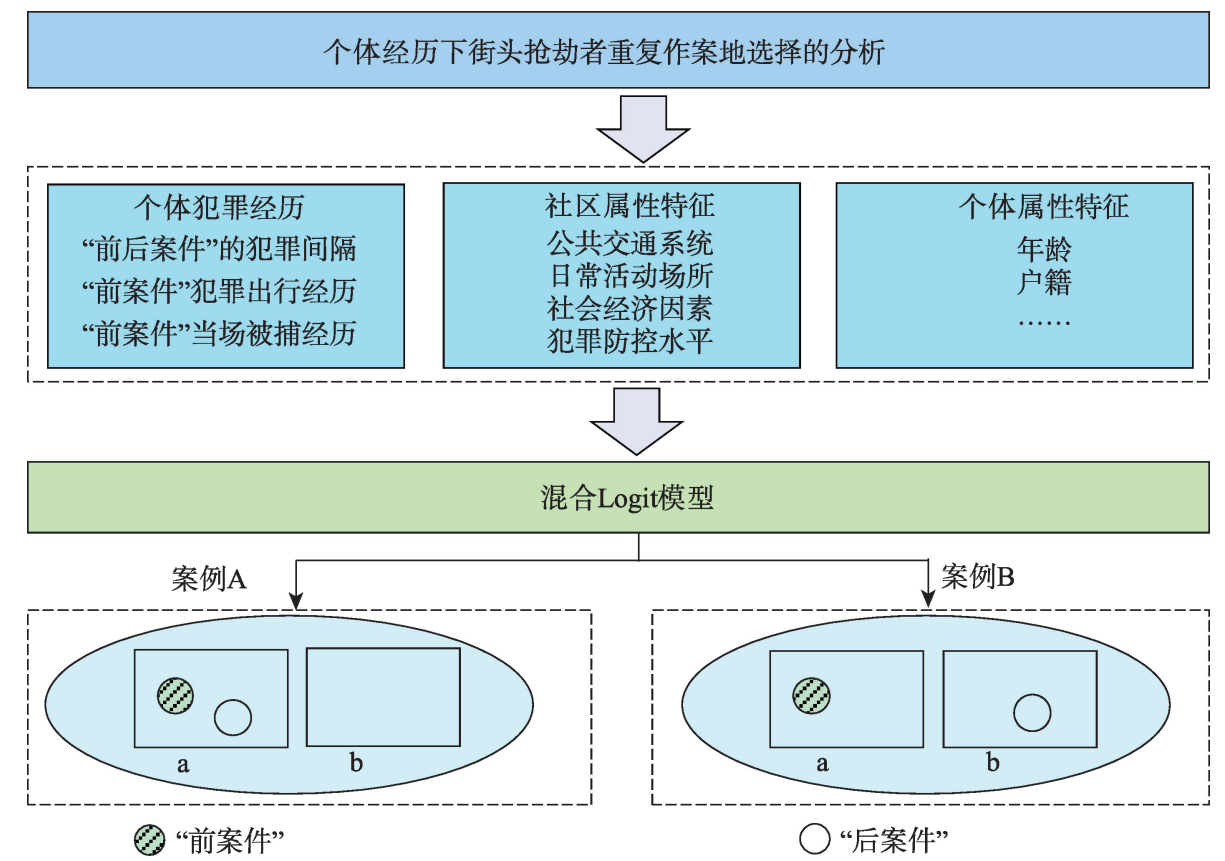

注:案例 $\mathrm{A}$ 表示“前案件”作案地与“后案件”作案地发生在相同的社区 $\mathrm{a}$ 中; 而案例 B 表示“前案 件”作案地与“后案件”作案地发生在不同的社区中,如社区 $\mathrm{a}$ 和社区 $\mathrm{b}$ 中。

图 1 街头抢劫者重复作案地选择的概念框架

Fig.1 Conceptual framework for street robbers' choice of repeated crime location 
素: 街头抢劫者的个体犯罪经历、个体属性特征和 作案地的社区属性特征。在这一概念框架中, 街头 抢劫者的个体犯罪经历包括以下 3 个方面: (1) “前 后案件” 的犯罪间隔; (2) “前案件”犯罪出行经历(用 犯罪出行距离刻画); (3) “前案件” 当场被捕经历。

可见, 先前的抢劫在一定程度上代表了积极的 学习经历。因此, 对重复犯罪的街头抢劫者来说, 我们认为如果 “前后案件”的犯罪间隔更小、“前案 件”犯罪出行距离更短, 以及在 “前案件” 中当场被 逮捕的可能性越小, 那么他们返回到“前案件” 区域 作案的可能性更大。“前后案件” 的犯罪间隔(或称 之为近因)是非常重要的, 因为在短时间内, 犯罪者 获得的信息可能是更准确的, 与之对应的环境要素 在这一时间内不可能有太大改变 ${ }^{[43-44]}$, 因此他们的 决策准则是保持稳定的。“前案件”中的犯罪出行距 离可能起到重要的作用, 对街头抢劫者来说, 他们 对其居住地附近的区域更熟悉, 进而更短的出行距 离可能代表更多的学习经历 ${ }^{[10,24]}$ 。“前案件” 当场被 捕与否是指特定时刻内犯罪者的个体经历, 也就是 说, 在行动中的被捕具有直接的消极结果, 而在行 动中未被捕却是延迟的消极结果。类似地, 犯罪被 捕的减少大大增加了犯罪的发生率 ${ }^{[4]}$ 。Benson ${ }^{[46]}$ 发 现了抓捕的可能性代表警察行动对犯罪产生的威 慑作用, 这对犯罪者的犯罪行为具有负面的影响。

需要指出的是, 以上概念框架中的社区属性特 征也是街头抢劫者作案地选择的准则, 主要包括作 案地的收益、成本和风险等指标。在本文的研究中, 这些指标没有明确地表述为假设, 但与个体属性特 征一样以控制变量的形式在模型中被测试, 因为其 不是本文所关注的焦点。总的来说, 除了社区相关 变量和个体属性特征之外, 本文假设街头抢劫者的 “后案件”作案地选择也受其个体犯罪经历的影响, 包括前后抢劫的犯罪间隔经历、先前抢劫的犯罪出 行经历, 以及在先前抢劫中未当场被捕经历。

\section{3 研究假设}

将以上 3 组变量纳人到混合 Logit模型中, 以评 估街头抢劫者先前的犯罪经历对其后续作案地选 择的影响。同时, 也将随机截距纳人模型中进行估 算。具体而言, 本文提出以下 3 个假设:

假设 1 : 对一个街头抢劫者来说, 如果“前后案 件”的犯罪间隔越临近, 那么“后案件” 作案地更可 能发生在他们“前案件”所在的区域。

犯罪间隔(时间间隔)是指“前案件”与 “后案件” 之间的时间差。重复犯罪的研究表明, 较短时间内
对同一目标或临近目标实施犯罪的往往是同一犯 罪者 ${ }^{[15,47-48]}$ 。随后在重复犯罪的少许研究中 ${ }^{[10-11]}$, 也 发现了“前后案件”时间越临近, “后案件”更可能发 生在先前的犯罪区域。由此, 本文认为街头抢劫者 “后案件”作案地选择“前案件”区域的可能性随着 时间的增长而降低。

假设 2 :对一个街头抢劫者来说, 如果“前案件” 中的犯罪出行距离越短, 那么“后案件”作案地更可 能发生在他们“前案件”所在的区域。

犯罪出行距离是指犯罪者居住地到作案地之 间的距离 ${ }^{[9-50]}$,参考已有研究 ${ }^{[51-53]}$, 以欧几里德距离 表示。假设 2 基于犯罪出行的视角考虑“前案件”的 犯罪经历对“后案件”作案地选择的影响。Lammers 等 ${ }^{[10]}$ 认为犯罪者的居住地靠近或包围他们的犯罪 目标。Ackerman 等 ${ }^{[50]}$ 利用地理画像发现了犯罪者 的出行距离具有分段函数的曲线衰减特征。这就 是说, 犯罪者对潜在目标的搜索, 存在一个明显的 距离衰减效应。无论在个体水平还是整体水平, 这 种效应表明犯罪者不太可能在远离他们居住地的 地方犯罪 ${ }^{[1,53-54]}$ 。换句话说, 通常情况下他们的犯罪 出行距离较短。由此, 本文认为街头抢劫者在 “前 案件”中的犯罪出行距离对他们“后案件”作案地选 择具有影响。

假设 3 : 对一个街头抢劫者来说, 与当场被捕的 “前案件” 区域相比,那么“后案件”作案地更可能发 生在他们未当场被捕的“前案件”区域。

街头抢劫发生在室外, 隐蔽性低, 当场被捕风 险较大。Cornish 等 ${ }^{[5]}$ 认为犯罪者的未来作案地选 择会受他们先前犯罪结果(被捕)的影响。Bernasco 等 ${ }^{[11]}$ 认为犯罪者决定在何处犯罪时,担心被捕可能 不是一个决定性因素,并提出“前案件”后的被捕不 影响他们“后案件”作案地选择的观点。然而, Lammers 等 ${ }^{[10]}$ 提出了犯罪者更可能重返他们先前未被 捕犯罪区域的观点。由此, 本文认为理性的街头抢 劫者很可能会把 “前案件”中的当场被捕看作是失 败经历, 并从中吸取经验与教训, 对他们“后案件” 作案地选择可能会产生影响。

\section{2 研究数据与方法}

\section{1 研究区域与数据集}

ZG 市位于中国大陆南方、珠江三角洲的北缘, 是国家中心城市和粤港澳大湾区的核心。长期以 来, $\mathrm{ZG}$ 市一直是华南地区的政治、经济、科教和文 
化中心。截至 2016 年, ZG 市辖 XY、WL、ZH、HT、 YB、PH、YP、DH、SN、CZ、HC 11 个区, 总面积 $7434.40 \mathrm{~km}^{2}$, 共设 136 个街道办事处、34个镇。同 时, ZG 市是一个超大城市, 作为中国东部沿海改革 开放的前沿城市, 其经济高速发展, 已成为中国经 济最活跃的城市之一。2016年ZG市实现地区生产 总值 1.96 万亿元, 人均 GDP 达到 1.42 万元, 分别比 2015 年增长 $8.0 \%$ 和 $4.2 \%$ 。考虑到 $\mathrm{SN} 、 \mathrm{CZ} 、 \mathrm{HC}$ 属 ZG 市的外围地区(远离市中心, 森林与农用地较 多), 因此, 本文以 ZG市 8 区下的 1973 个社区为研 究区(图2), 其中, 这些社区面积的标准差为 2.85 , 平 均面积 $1.62 \mathrm{~km}^{2}$, 最小面积为 $0.001 \mathrm{~km}^{2}$, 最大面积为 $31.43 \mathrm{~km}^{2}$ 。

\subsection{1 犯罪数据}

数据来源于 ZG 市公安局提供的 2012-2016年 ZG市的街头抢劫者抓捕数据。类似已有的研究 ${ }^{[10-11]}$, 该类数据是指已经向警方报案, 且至少有一个街头 抢劫者被抓获。该类数据记录了街头抢劫者详细 的个体信息及其案件信息。个体信息如街头抢劫 者的姓名、性别、民族、户籍地址、出生日期、文化程 度以及吸毒与否等属性; 案件信息如抢劫时间、抢 劫地址和抢劫时的居住地, 以及犯罪对象、犯罪场
所、作案工具、作案手段和损失财物或金额等。同 时, ZG 市公安局已通过对被捕者的审问及案件连 锁分析,侦破了之前立案但当时未将他们抓获的案 件。因此,通过比对街头抢劫者的姓名、出生日期、 户籍地址等个人信息, 可以识别出重复的(抢劫 2 次 以上)街头抢劫者, 也可以匹配出他们重复犯罪下 的“前案件”信息和“后案件”信息。此外,本文也考 虑了街头抢劫者作案时的居住地。

该数据共有 11455 条警察记录, 排除了街头抢 劫者居住地在研究区外的 2285 条记录, 以及 1310 条无固定住所或居住地不明的记录,剩下 7860 个记 录, 涉及 7124 个街头抢劫者。而对于团伙抢劫案件 (1起案件涉及 2 个或 2 个以上的抢劫者), 通过比对 个体信息和案件信息, 则可识别出团伙抢劫案件与 单独抢劫案件, 从而确定了街头抢劫案件的数量 为 4358 起(表 1)。与此同时, 对于涉及团伙抢劫案 件的犯罪者, 本文参考已有文献 ${ }^{[3,8]}$, 从中随机选取 一个街头抢劫者作为研究的部分样本 (1712个), 最 终有 4358 个街头抢劫案件纳人到重复样本构建中 (表1)。

从理论上来看, 抢劫 1 次的犯罪者没有先前的 个体经历, 鉴于此, 本文焦聚于抢劫 $n$ 次的犯罪者。

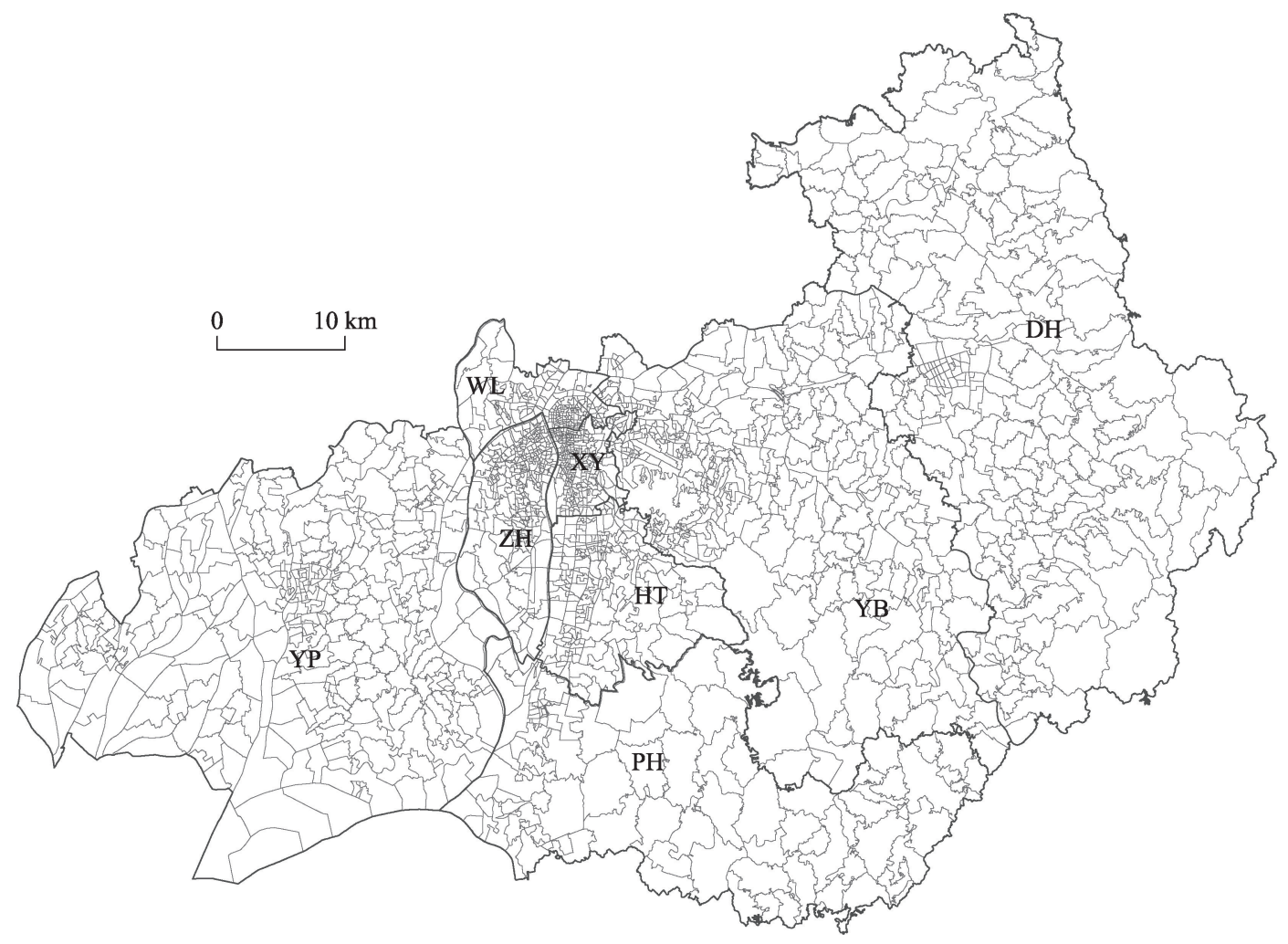

图 2 研究区示意图(ZG市 8区)

Fig.2 Schematic map of the research area (8 districts of ZG City) 
表 1 基于抓捕数据的街头抢劫案件基本情况 $(N=4358)$

Tab.1 Basic situation of street robbery cases based on arrest data $(N=4358)$

\begin{tabular}{cccc}
\hline 涉案人数/人 & 被捕记录/次 & 案件数/起 & 案件比例/\% \\
\hline 1 & 2646 & 2646 & 60.72 \\
2 & 1758 & 879 & 20.17 \\
3 & 1209 & 403 & 9.25 \\
4 & 812 & 203 & 4.66 \\
5 & 565 & 113 & 2.59 \\
$\geqslant 6$ & 870 & 114 & 2.62 \\
合计 & 7860 & 4358 & 100 \\
\hline
\end{tabular}

如利用每个街头抢劫者的唯一标识符, 可以识别出 重复的犯罪者(抢劫 2 次或 2 次以上), 即通过比对街 头抢劫者姓名、出生日期、户籍所在地等个人身份 信息, 进而识别出重复的街头抢劫者, 并匹配出他 们重复犯罪下的“前案件”信息和“后案件”信息,同 时也考虑了街头抢劫者作案时的居住地。对团伙 抢劫者也按以上的方式处理。例如,一个“前后案 件” 是由抢劫者 $\mathrm{A}$ 、抢劫者 $\mathrm{B}$ 和抢劫者 $\mathrm{C}$ 实施的, 那 么就随机抽取一个抢劫者去构建样本。最后, 篮选 出 527 个重复犯罪的街头抢劫者,涉及 1262 起街头 抢劫案件。该样本的个体属性特征如性别和年龄 见表 2 所示。例如, 重复犯罪的街头抢劫者几乎为 男性 (520人), 所占比例为 $98.67 \%$, 而女性仅为 7 人, 所占比例为 $1.33 \%$ 。同时, 这些抢劫者在年龄上绝 大多数是青少年和成年人(12 50 岁, 96.77\%), 51 岁 及以上的抢劫者占少数 $(3.23 \%)$ 。

从表 3 可以看出, 重复犯罪的街头抢劫者实施 的抢劫案件次数如下: 涉及 2 起案件的重复抢劫者 为 412 人,所占比例为 $78.18 \%$; 涉及 3 起案件的重复 抢劫者为 69 人,所占比例为 $13.09 \%$; 涉及 4 起案件 的重复抢劫者为 21 人,所占比例为 $3.98 \%$; 大于或
等于 5 起的比例为 $4.75 \%$ 。例如,一个重复抢劫者 涉及 2 起抢劫案件, 就有 1 对“前后案件”; 一个重复 抢劫者涉及 3 起抢劫案件, 就有 3 对“前后案件”,包 括第 1 次和第 2 次,第 2 次和第 3 次,第 1 次和第 3 次。以此类推, 如果一个重复抢劫者涉及 $n$ 件案件, 那么就有 $n(n-1) / 2$ 对“前后案件”。最后匹配的前一 后街头抢劫案件共 1118 起(表 3 )。

\subsection{2 观测变量}

通过构建多个变量来检验上述假设。首先, 创 建一个“重复地”变量, 用以表达街头抢劫者的“前 案件”抢劫地与其“后案件”抢劫地是否相同。如果 “前案件”抢劫地与其“后案件”抢劫地在同一个社 区, 那么“重复地”变量就记为“1”(692 起,或 62\%), 否则记为“ 0 ”(426起,或 38\%)。

第二, “前后案件”的犯罪间隔变量, 是指“前后 案件”发生的时间差值, 以“前案件”和“后案件”之 间的天数进行测算(表 4)。类似已有的研究 ${ }^{[3,8]}$, 本文 包括以下 6 个不同的间隔类别: (1) 0 2 d; (2) 3 7 d; (3) $8 \sim 30 \mathrm{~d}$; (4) 1 6个月; (5) 7 24 个月; (6) 大于 24 个

表 2 重复街头抢劫者的个体属性特征

Tab.2 Characteristics of the street robbers

\begin{tabular}{lcc}
\hline \multicolumn{1}{c}{ 个体属性 } & 数量/人 & 百分比 $/ \%$ \\
\hline 性别 & & \\
男性 & 520 & 98.67 \\
女性 & 7 & 1.33 \\
年龄(截至2016年12月) & & \\
12 18岁 & 58 & 11.01 \\
19 23岁 & 152 & 28.84 \\
$24 \sim 30$ 岁 & 137 & 26.00 \\
31 40岁 & 109 & 20.68 \\
$41 \sim 50$ 岁 & 54 & 10.25 \\
51 岁及上上 & 17 & 3.23 \\
\hline
\end{tabular}

表 3 重复街头抢劫案件的匹配数

Tab.3 Pairs of prior and subsequent street robberies

\begin{tabular}{|c|c|c|c|c|c|c|}
\hline \multirow{2}{*}{$\begin{array}{c}\text { 重复抢劫 } \\
\text { 次数 }\end{array}$} & \multicolumn{2}{|c|}{ 街头抢劫者 } & \multicolumn{2}{|c|}{ 街头抢劫案件 } & \multicolumn{2}{|c|}{ 匹配案件 } \\
\hline & 数量/人 & 百分比/\% & 数量/起 & 百分比/\% & 数量/起 & 百分比 $/ \%$ \\
\hline 2 & 412 & 78.18 & 824 & 65.29 & 412 & 36.85 \\
\hline 3 & 69 & 13.09 & 207 & 16.40 & 207 & 18.52 \\
\hline 4 & 21 & 3.98 & 84 & 6.66 & 126 & 11.27 \\
\hline 5 & 13 & 2.47 & 65 & 5.15 & 130 & 11.63 \\
\hline 6 & 4 & 0.76 & 24 & 1.90 & 60 & 5.37 \\
\hline 7 & 7 & 1.33 & 49 & 3.88 & 147 & 13.15 \\
\hline 9 & 1 & 0.19 & 9 & 0.71 & 36 & 3.22 \\
\hline 合计 & 527 & 100 & 1262 & 100 & 1118 & 100 \\
\hline
\end{tabular}


表 4 重复街头抢劫案件的犯罪间隔 $(N=1118)$

Tab.4 Time interval of prior and subsequent street robberies $(N=1118)$

\begin{tabular}{lcc}
\hline 犯罪间隔 & 案件数/起 & 百分比 $/ \%$ \\
\hline $0 \sim 2 \mathrm{~d}$ & 210 & 18.78 \\
$3 \sim 7 \mathrm{~d}$ & 123 & 11.00 \\
$8 \sim 30 \mathrm{~d}$ & 234 & 20.93 \\
$1 \sim 6$ 个月 & 205 & 18.34 \\
$7 \sim 24$ 个月 & 241 & 21.56 \\
大于 24 个月 & 105 & 9.39 \\
\hline
\end{tabular}

月。例如,一个重复犯罪者实施了 2 次抢劫, 其“前 案件” 和“后案件”的犯罪间隔为 $5 \mathrm{~d}$, 那么“ “ $3 \sim 7 \mathrm{~d}$ ”的 间隔类别就记为 “ 1 ”, 而其他类别均记为 “ 0 ”。在 “0 2 d” 的犯罪间隔中, 有 172 对 “后案件”与“前案 件”发生在相同的社区中, 而38对发生在不同的社 区中。与以上类似, “3 7 d” 依次为 98 对和 25 对、 “8 30 d”依次为 171 对和 63 对、“1 6个月”依次为 124 对和 81 对、“7 24 个月”依次为 90 对和 151 对、 “大于 24 个月”依次为 37 对和 68 对。

第三, “前案件”犯罪出行距离变量, 是指街头 抢劫者在 “前案件” 中的居住地到作案地之间的距 离, 以欧几里德距离表示, 其均值为 $4.20 \mathrm{~km}$ 。已有 研究表明, “前案件”作案地的吸引力与该距离具有 指数函数关系 ${ }^{[15]}$ 。因此, 为了使其线性化, 在本文 的模型中使用了该距离的对数值。最后, “前案件” 未当场被捕变量, 用以检验街头抢劫者“前案件”被 捕经历是否影响其“后案件” 作案地的选择。如果 一个街头抢劫者在“前案件”中未当场被捕(在行动 中或逃逸过程中未被捕), 那么这一变量就记为“1” (872 起, 或 78\%), 反之记为“0”(246 起, 或 $22 \%)$, 该 变量的均值为 0.78 。在未当场被捕的情况下, “前 后案件”的作案地发生在同一社区的数量为 629 起, 在不同社区的数量为 243 起; 而在当场被捕的情况 下，“前后案件”的作案地发生在同一社区的数量为 22 起, 在不同社区的数量为 224 起。

\subsection{3 控制变量}

已有文献验证了犯罪者作案地选择的主要影 响因素有: (1) 公共交通系统, 如公交站、客运站、地 铁站、道路网络等 ${ }^{[3,756-57]}$; (2) 日常活动场所, 如酒吧 与会所、网吧、商场与超市、银行与 ATM、餐馆与住 宿以及中学等 ${ }^{[8,21,58-59]}$; 3 社会经济因素, 如种族分 异、外来人口比重、青少年人口比重、单亲家庭居民

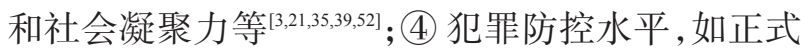
和非正式控制、破案率和热点警务等 ${ }^{[1934,60-63]}$ 。因此,
结合已有研究和 ZG 市的实际情况, 本文考虑选取 的控制变量包括:公交站、客运站、地铁站、道路网 络密度、商场与超市、杂货店、批发市场、酒吧与会 所、中学、外来人口比重、青少年人口比重、破案率、 “后案件”出行距离, 用街头抢劫者“后案件”作案地 到其居住地之间的欧几里德距离的对数值表示, 以 及将街头抢劫者的年龄和户籍 2 类个体属性融人混 合 Logit模型中, 其中年龄变量采用实际年龄值, 而 户籍变量采用虚拟变量“ 1 ”和“ 0 ”,分别代表外来户 籍和本地户籍。除外来人口比重、青少年人口比重 及破案率(社区中已破案数除以该类型案件数)外, 其他数据均采取 2016 年 ZG 市 POI 数据, 该数据来 源于中国某空间信息服务公司,其数据类型为点矢 量数据, 如公交站、客运站、地铁站以及商场与超 市、中学等变量的数值均为各个社区内相应 POI 的 数量。因此, 可以利用 ArcGIS 10.3 软件空间连接 功能聚合到对应的社区中去。

\section{2 混合 Logit 模型}

混合 Logit模型(Mixed Logit Model)是离散选 择模型的新发展 ${ }^{[6]}$, 具有较高的灵活性 ${ }^{[6]}$, 适合任何 形式的分布 ${ }^{[66]}$, 被认为是离散选择模型的精细化 ${ }^{[16]}$ 。 混合 Logit 模型能评估决策者从互斥、完备和有限 的选项集中选择其中一个的概率, 这种概率是由决 策者的特征、选项集和未观测到的因素所决定的， 因而混合Logit 模型被广泛应用于分析微观经济学 和社会科学中的离散选择行为。考虑到作案地选 择与上述选择类似, 因此, Townsley 等 ${ }^{[16]}$ 认为这种 统计模型特别适合于犯罪者的面板数据, 因为这种 评估考虑了犯罪案件嵌套于犯罪者的问题。

同时, 混合 Logit模型是条件 Logit模型更一般 化的版本 ${ }^{[10-11]}$ 。最近,一些研究利用该方法对犯罪 者的作案地选择进行了分析。例如, Townsley 等 ${ }^{[16]}$ 检验了澳大利亚布里斯班住宅窃贼的作案地选择; 随后, Frith 等 ${ }^{[67]}$ 检验了英国海威康市人室盗窃者的 作案地选择。这些文献讨论了犯罪者的作案地选 择如何受一系列因素的影响,这些因素包括但不限 于犯罪场所、人类活动及其出行距离。

在控制街头抢劫者个体属性特征的前提下, 本 文假定街头抢劫者犯罪时将会选择对其效应最大 的社区。效应函数计算公式如下:

$$
U_{i j}=\beta_{i} x_{i j}+\varepsilon_{i j}
$$

式中: $U_{i j}$ 为街头抢劫者 $i$ 选择在社区 $j$ 抢劫的预期效 应; $x_{i j}$ 为街头抢劫者 $i$ 和社区 $j$ 相关的解释变量的 
值; $\beta_{i}$ 为街头抢劫者 $i$ 的系数估计向量; $\varepsilon_{i j}$ 为模型的 随机误差。

效用函数可以根据混合 Logit 模型估计得到。 因此, 街头抢劫者 $i$ 选择在社区 $j$ 抢劫概率的计算公 式如下:

$$
L_{i j}\left(\beta_{i}\right)=\frac{\mathrm{e}^{\beta_{i} x_{i j}}}{\sum_{k} \mathrm{e}^{\beta_{x} x_{i k}}}
$$

通过对式(2)中 $\beta_{i}$ 的所有取值进行积分转换成 $L_{i j}\left(\beta_{i}\right)$, 可以得到无条件选择的概率:

$$
\operatorname{Prob}\left(Y_{i}=j\right)=\int L_{i j}\left(\beta_{i}\right) f(\beta \mid \theta) \mathrm{d} \beta
$$

式中: $Y_{i}$ 为街头抢劫者 $i$ 的选择; $\theta$ 为密度函数 $\beta_{i}$ 的参 数(如均值、标准差和方差等); 无条件选择概率(混 合 Logit 概率) 是基于 $\beta_{i}$ 值计算的 Logit 概率加权平 均的积分, 这个权重是由密度函数 $f(\beta \mid \theta)$ 所决定。 本文基于 STATA 13.0 软件, 采用最大似然法进行模 型计算。

\section{3 结果分析}

首先, 对自变量和控制变量进行多重共线性检 验。结果表明公交站与道路网络密度的相关系数最 大,为 0.49 , 其VIF 值为 1.56 , 但远小于 10 。同时, 这 些变量的 VIF 平均值为 1.75 , 说明了这些变量之间 没有显著的共线性,能适用于混合 Logit模型分析。

混合 Logit模型的评估结果主要包括: 优势比 (Odds Ratios, OR)、标准误差(Standard Error, SE)、Z 值、显著性水平、置信区间、伪 $R^{2}$ 等 (表 5 、表 6 、表 7)。为便于解释, 本文将对 3 个模型作简要介绍, Model 1 Model 3 分别检验街头抢劫者“前案件”中 的犯罪间隔、犯罪出行距离和未当场被捕的个体犯 罪经历对其“后案件”作案地选择的影响。同理,需 要说明的是模型的整体拟合度,与离散选择模型一 样, 混合 Logit 模型的伪 $R^{2}$ 通常比普通最小二乘法

表 5 基于混合 Logit 模型的假设 1 检验结果(Model 1)

\begin{tabular}{|c|c|c|c|c|}
\hline 变量 & OR & SE & $Z$ & $95 \%$ 的置信区间 \\
\hline \multicolumn{5}{|l|}{ “前后案件” 的犯罪间隔 } \\
\hline $0 \sim 2 \mathrm{~d}$ & $24.453^{* * * *}$ & 16.442 & 4.75 & {$[6.546,91.345]$} \\
\hline $3 \sim 7 \mathrm{~d}$ & $17.928^{* * *}$ & 12.541 & 4.13 & {$[4.551,70.623]$} \\
\hline $8 \sim 30 \mathrm{~d}$ & $9.007^{* *}$ & 5.885 & 3.36 & {$[2.503,32.412]$} \\
\hline $1 \sim 6$ 个月 & $7.870^{* *}$ & 5.292 & 3.07 & {$[2.107,29.399]$} \\
\hline 7 24个月 & 1.943 & 1.284 & 1.01 & {$[0.532,7.093]$} \\
\hline 大于 24 个月 & 1.000 & 1.000 & 1.000 & {$[1.000,1.000]$} \\
\hline 公交站 & $1.071^{* *}$ & 0.026 & 2.84 & {$[1.021,1.122]$} \\
\hline 客运站 & $1.621^{* *}$ & 0.260 & 3.01 & {$[1.184,2.219]$} \\
\hline 地铁站 & $0.855^{*}$ & 0.060 & -2.21 & {$[0.745,0.982]$} \\
\hline 道路网络密度 & $0.932^{*}$ & 0.035 & -2.09 & {$[0.866,0.997]$} \\
\hline 商场与超市 & 0.994 & 0.016 & -0.38 & {$[0.964,1.025]$} \\
\hline 杂货店 & 0.975 & 0.038 & -0.64 & {$[0.903,1.053]$} \\
\hline 批发市场 & 0.959 & 0.043 & -0.93 & {$[0.879,1.047]$} \\
\hline 酒吧与会所 & $1.091^{*}$ & 0.050 & 3.12 & {$[1.037,1.192]$} \\
\hline 中学 & $1.240^{*}$ & 0.133 & 2.00 & {$[1.004,1.530]$} \\
\hline 外来人口比重 & 1.143 & 0.377 & 0.40 & {$[0.598,2.183]$} \\
\hline 青少年人口比重 & 0.688 & 0.408 & -0.63 & {$[0.216,2.196]$} \\
\hline 破案率 & 1.693 & 1.712 & 0.52 & {$[0.233,12.284]$} \\
\hline “后案件”犯罪出行距离 & $0.163^{* * *}$ & 0.042 & -7.00 & {$[0.098,0.271]$} \\
\hline 年龄 & 1.028 & 0.019 & 1.54 & {$[0.992,1.065]$} \\
\hline 户籍 & $2.184^{*}$ & 1.055 & 2.04 & {$[1.048,5.628]$} \\
\hline 常数项 & $0.005^{* * * *}$ & 0.005 & -5.13 & {$[0.001,0.036]$} \\
\hline 随机效应参数 & 估计值 & 标准误差 & & 95\%的置信区间 \\
\hline 标准差(常数项) & 1.647 & 0.304 & & {$[1.147,2.365]$} \\
\hline
\end{tabular}

Tab.5 Test result of hypothesis 1 based on amixed logit model (Model 1)

注: ***、**分别表示 $P<0.05 、 P<0.01 、 P<0.001$,下同; 模型 $P$ 值 $\rightarrow 0$; 伪 $R^{2}=0.245$ 。 
表 6 基于混合 Logit 模型的假设 2 检验结果(Model 2)

Tab.6 Test result of hypothesis 2 based on a mixed logit model (Model 2)

\begin{tabular}{|c|c|c|c|c|}
\hline 变量 & OR & SE & $Z$ & 95\%的置信区间 \\
\hline “前案件”犯罪出行距离 & $0.599^{*}$ & 0.151 & -2.04 & {$[0.366,0.981]$} \\
\hline 公交站 & $1.078^{* * *}$ & 0.029 & 2.78 & {$[1.022,1.136]$} \\
\hline 客运站 & $1.565^{* *}$ & 0.268 & 2.62 & {$[1.119,2.190]$} \\
\hline 地铁站 & $0.864^{*}$ & 0.065 & -2.11 & {$[0.745,0.986]$} \\
\hline 道路网络密度 & $0.902^{*}$ & 0.037 & -2.49 & {$[0.832,0.978]$} \\
\hline 商场与超市 & 0.981 & 0.017 & -1.10 & {$[0.949,1.015]$} \\
\hline 杂货店 & 0.978 & 0.041 & -0.54 & {$[0.901,1.061]$} \\
\hline 批发市场 & 0.934 & 0.046 & -1.39 & {$[0.849,1.028]$} \\
\hline 酒吧与会所 & $1.088^{*}$ & 0.054 & 2.98 & {$[1.027,1.200]$} \\
\hline 中学 & 1.205 & 0.138 & 1.63 & {$[0.964,1.507]$} \\
\hline 外来人口比重 & 1.257 & 0.449 & 0.64 & {$[0.624,2.533]$} \\
\hline 青少年人口比重 & 0.543 & 0.343 & -0.97 & {$[0.158,1.871]$} \\
\hline 破案率 & 4.884 & 5.160 & 1.50 & {$[0.616,38.739]$} \\
\hline “后案件”犯罪出行距离 & $0.158^{* * * *}$ & 0.048 & -6.11 & {$[0.087,0.286]$} \\
\hline 年龄 & 1.010 & 0.020 & 0.51 & {$[0.972,1.050]$} \\
\hline 户籍 & $2.318^{*}$ & 1.220 & 2.16 & {$[1.086,6.504]$} \\
\hline 常数项 & $0.049^{* * * *}$ & 0.043 & -4.04 & {$[0.002,0.079]$} \\
\hline 随机效应参数 & 估计值 & 标准误差 & & 95\%的置信区间 \\
\hline 标准差(常数项) & 2.055 & 0.383 & & {$[1.426,2.962]$} \\
\hline
\end{tabular}

注:模型 $P \rightarrow 0$; Pseudo $R^{2}=0.192$ 。

表 7 基于混合 Logit 模型的假设 3 检验结果(Model 3)

Tab.7 Test result of hypothesis 3 based on a mixed logit model (Model 3)

\begin{tabular}{|c|c|c|c|c|}
\hline 变量 & $\mathrm{OR}$ & SE & $Z$ & 95\%的置信区间 \\
\hline “前案件”未当场被捕 & $10.029^{* * * *}$ & 4.783 & 4.83 & {$[3.938,25.538]$} \\
\hline 公交站 & $1.074^{* *}$ & 0.029 & 2.66 & {$[1.019,1.132]$} \\
\hline 客运站 & $1.860^{* *}$ & 0.345 & 3.35 & {$[1.293,2.676]$} \\
\hline 地铁站 & $0.867^{*}$ & 0.069 & -2.06 & {$[0.742,0.990]$} \\
\hline 道路网络密度 & $0.914^{*}$ & 0.037 & -2.19 & {$[0.844,0.990]$} \\
\hline 商场与超市 & 0.998 & 0.017 & -0.14 & {$[0.964,1.032]$} \\
\hline 杂货店 & 0.979 & 0.042 & -0.50 & {$[0.901,1.064]$} \\
\hline 批发市场 & 0.930 & 0.045 & -1.49 & {$[0.845,1.023]$} \\
\hline 酒吧与会所 & $1.078^{*}$ & 0.054 & 2.13 & {$[1.017,1.190]$} \\
\hline 中学 & 1.234 & 0.142 & 1.83 & {$[0.985,1.545]$} \\
\hline 外来人口比重 & 1.237 & 0.448 & 0.59 & {$[0.608,2.517]$} \\
\hline 青少年人口比重 & 0.608 & 0.391 & -0.77 & {$[0.172,2.143]$} \\
\hline 破案率 & 1.838 & 1.965 & 0.57 & {$[0.226,14.942]$} \\
\hline “后案件”犯罪出行距离 & $0.119^{* * * *}$ & 0.036 & -6.97 & {$[0.065,0.216]$} \\
\hline 年龄 & 1.005 & 0.020 & 0.26 & {$[0.967,1.045]$} \\
\hline 户籍 & $3.239^{*}$ & 1.739 & 2.19 & {$[1.131,9.276]$} \\
\hline 常数项 & $0.007^{* * *}$ & 0.007 & -4.86 & {$[0.001,0.051]$} \\
\hline 随机效应参数 & 估计值 & 标准误差 & & 95\%的置信区间 \\
\hline 标准差(常数项) & 2.006 & 0.384 & & {$[1.378,2.921]$} \\
\hline
\end{tabular}

注: 模型 $P \rightarrow 0$; Pseudo $R^{2}=0.219$ 。

的 $R^{2}$ 低很多。McFadden ${ }^{[6]}$ 已证实了伪 $R^{2}$ 大于 0.20 , 就可以认为该模型具有完美的拟合度。此外,混合
Logit模型的 $P$ 值表示随机截距模型与固定效应 Logistic 回归模型相比的显著改进程模型度, 如果 $P$ 值 
接近 0 ,说明该模型的改进效果就越明显 ${ }^{[69]}$ 。

在表 5 中,变量的 OR 值大于 1 表示正的影响, 它越大表示正影响越大; OR 值小于 1 表示负的影 响, 它越小表示负影响越大。例如, 在 Model 1 中客 运站的 OR 值为 1.621 , 这是一个正效应: 表示如果 “前案件”社区内的客运站每增加 1 个, 那么街头抢 劫者“后案件”作案地选择“前案件”社区的可能性 就会增加 $62.1 \%$ 。此外, 该变量 95\%置信区间的下 限和上限分别为 1.184 和 2.219 , 因此, 这种影响的 差异具有统计学意义。

本文首先讨论“前后案件”犯罪间隔的影响。 Model 1 的结果验证了假设 1 的成立, 该模型的伪 $R^{2}$ 为 $0.245, P$ 值趋近 0 , 这说明了该模型具有较好的解 释能力, 并有明显的改进。从表 5 中可以看出, 前 5 类“前后案件”的犯罪间隔的 OR 值均大于 1 , 除“7 24 个月”这一间隔类别的 $P$ 值大于 0.05 , 其他类别 的犯罪间隔的 $P$ 值均小于 0.01 (如 $0 \sim 2 \mathrm{~d}$ 和 $3 \sim 7 \mathrm{~d}, P<$ $0.001 ; 8 \sim 30 \mathrm{~d}$ 和 1 6个月, $P<0.01)$, 这说明了“前后 案件”的犯罪间隔变量在前 4 类间隔类别上的影响 是积极的, 且在统计学上是非常显著的。也就是 说, 如果“前后案件”的发生时间越临近, 那么街头 抢劫者“后案件”作案地更可能发生在他们“前案 件”所在的区域。

从“前后案件”的犯罪间隔变量的影响来看, 街 头抢劫者更可能在他们先前犯罪过的区域再次抢 劫。此外, 随着 “前后案件” 犯罪间隔的增加, OR 值 急剧下降。与过去 $2 \mathrm{a}$ 前没有抢劫过的社区相比, 他们选择过去 $2 \mathrm{~d}$ 内抢劫过的社区的可能性会增加 24.453 倍, 选择过去 3 7 d 内抢劫过的社区的可能 性会增加 17.928 倍, 选择过去 8 30 d 内抢劫过的社 区的可能性会增加 9.007 倍, 选择过去 1 6个月内抢 劫过的社区的可能性会增加 7.870 倍。街头抢劫者 选择过去 7 24 个月抢劫过的社区的可能性虽无显 著的统计学意义,但仍比过去 24 个月没有抢劫过的 社区高 1.943 倍。因此, 街头抢劫者“后案件”作案 地选择“前案件” 区域的可能性随着时间的增长而 降低。

从表 6 中可知, Model 2 验证了假设 2 , 即街头 抢劫者“前案件”的地点对他们“后案件” 作案地选 择具有影响。根据 Model 2 的结果, 它的伪 $R^{2}$ 为 0.192 , “前案件”犯罪出行距离变量的 OR 值为 0.599 $(P<0.05)$, 这表明街头抢劫者“前案件” 中的犯罪出 行距离对他们“后案件”作案地选择具有显著的负 影响。因此, 这与本文的假设 2 一致: 当街头抢劫者
“前案件”犯罪出行距离越短时,那么他们后续作案 地越可能发生在“前案件”所在的区域。

与假设 3 一致, Model 3 验证了街头抢劫者在 “前案件”区域未当场被捕经历对他们“后案件”作 案地选择具有显著的影响。从表 7 可以看出, Model 3 的伪 $R^{2}$ 为 0.219 ( $P$ 趋近于 0$)$, 这说明该模型具有 较强的解释能力及进行了显著改进。同时, “前案 件”未当场被捕变量的 OR 值为 10.029 ( $P$ 趋近于 0$)$, 表明该变量的影响具有显著的正效应以及统计学 意义。这一结果表明,对同一个街头抢劫者来说, 他在未当场被捕的情况下,返回先前的犯罪区域的 可能性非常大。换句话说, 可能存在这样一种情 况, 即在行动中的被捕是一种直接的消极结果, 而 在行动中未被捕是一种延迟的消极结果。因此,这 一发现支持了第 3 个假设, 即街头抢劫者更有可能 返回到他们先前未当场被捕的“前案件” 区域实施 抢劫。

\section{4 结论与讨论}

本文通过融合犯罪模式理论、理性选择理论和 社会解组等理论,在已有研究的基础上,结合街头 抢劫者的个体犯罪经历对其重复作案地选择制定 了明确假设, 并以中国东南沿海特大城市 ZG 市为 例, 利用街头抢劫者的抓捕数据和混合 Logit模型, 深人检验了街头抢劫者“前案件”的个体犯罪经历 对他们“后案件”作案地选择的影响。研究发现: 街 头抢劫者在“前案件”中的犯罪间隔、犯罪出行距离 和当场被捕等个体犯罪经历对其“后案件”作案地 选择具有强烈的影响, 具体表现为,如果“前后案 件”的犯罪间隔越临近、“前案件”犯罪出行距离越 短, 以及“前案件”未当场被捕, 则大大增加了街头 抢劫者返回到先前抢劫区域再次犯罪的可能性。 通过警察访谈和定性分析,发现“前案件”未当场被 捕是由犯罪者当场被捕的恐惧感、警察特殊的干预 方式及社会凝聚力和犯罪防控的相互作用而形成。

本文的第一个发现,即街头抢劫者“前后案件” 的犯罪间隔越短,其“后案件”的作案地更可能发生 在他们“前案件”所在的区域,这一结果与 Bernasco 等 ${ }^{[11]}$ 和 Lammers 等 ${ }^{[10]}$ 的研究一致。这说明了街头抢 劫者“后案件”作案地选择“前案件”区域的可能性 随着时间的增长而降低。根据犯罪模式理论和理 性选择理论,随着时间演化和环境变化,犯罪者对 过去犯罪场所的熟悉程度(感知)可能会逐渐降低, 
而时间越临近的“前后案件”, 通常先前作案地的环 境要素不会发生较多变化。因此, 本文发现街头抢 劫者的犯罪间隔经历影响他们“后案件”作案地的 选择是合理的。

本文的第二个发现, 即街头抢劫者在“前案件” 中的犯罪出行距离越短, 其“后案件”作案地更可能 发生在他们“前案件”所在的区域,这一结果与已有的 研究一致 ${ }^{[2,53,70]}$ 。对犯罪者来说, 到一个陌生地方作 案, 这不仅会有暴露的风险, 而且有可能消耗或浪 费他们的有限资源, 特别是时间、金钱或精力 ${ }^{[5]}$ 。 根据犯罪模式理论和日常活动理论, 犯罪者倾向在 他们居住地及其附近区域去寻找合适的目标, 这不 仅有助于减少犯罪出行距离, 而且有利于找到金钱 汇集或现金交易的地方。此外,犯罪者受自身的生 活方式和日常活动的影响, 以他们习惯性的出行模 式为导向, 且很大程度上受限于他们所熟悉的感知 空间 ${ }^{[1]}$ 。因此, 本文发现街头抢劫者在“前案件”中 的犯罪出行经历影响他们 “后案件” 作案地选择也 是合理的。

本文的第三个发现与 Lammer 等 ${ }^{[10]}$ 的犯罪者更 可能返回到他们未被捕的“前案件” 区域犯罪的观 点类似, 也验证了街头抢劫者“后案件” 作案地选择

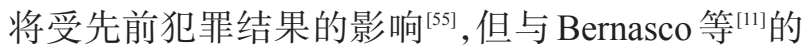
“前案件”后的被捕不影响其随后人室盗窃作案地 选择的观点不同。根据街头抢劫的案件特点和中 国的实际情况, 街头抢劫者“后案件” 作案地不太可 能选择他们当场被捕的“前案件” 区域的主要原因 有以下 3 点:第一, 当场被捕的恐惧感。在中国现实 社会中, 街头抢劫者如果当场被抓, 那么他们很可 能会遭到受害者及其亲人或朋友的报复。因此, 街 头抢劫者可能会对当场被捕的地方心存恐惧, 在他 们的内心可能会留下 “一朝被蛇咬, 十年怕井绳” 的 阴影。根据理论选择理论, 街头抢劫者将吸取“前 案件” 中当场被捕的惨痛教训, 并促使其以后的犯 罪将远离这一被捕区域。第二, 警察特殊的干预方 式。根据情景预防犯罪, 在中国城市社会治理背景 下,警察通常会采用“拍肩膀”方式(警察访谈, 内部 说法)对过去被捕的人进行提前干预。如果警察发 现在他们管辖范围内过去被捕的人再次出现时, 他 们通常会采取“上门查房” “招呼传唤” 等“拍肩膀” 方式对其干预, 对犯罪者给以特殊 “问候”, 也就是 警告他们不要在其管辖范围内犯罪。这种特殊的 干预方式, 再加上社会治安视频监控的面部识别能 力, 在一定程度上能迫使犯罪者的后续犯罪远离这
个先前被抓捕的区域。此外,中国人民警察通常会 设点盘查可疑人员的身份证。《中华人民共和国居 民身份证法》的第十五条赋予了人民警察依法执行 职务时可查验居民身份证的权力, 同时这也是公安 机关的一项日常执法活动。而 Lammers 等 ${ }^{[10]}$ 和 Bernasco 等 ${ }^{[1]}$ 研究区的国家法律没有赋予警察这种权 力。因此, 之前被逮捕的街头抢劫者再次出现在这 个区域时,一旦被辖区内的警察发现,他们将会受 到严密监控,如再次犯罪就有较高的被捕风险。因 此, 中国警察这种特殊的干预方式, 将迫使街头抢 劫者不太可能在先前被捕的“前案件” 区域再次犯 罪。第三,社会凝聚力和犯罪防控水平。街头抢劫 者在“前案件” 区域当场被捕, 这一定程度上反映了 该区域可能具有较强的社会凝聚力或较高的犯罪 防控水平。根据社会解组理论, 如社会凝聚力的存 在, 有利于居民之间形成集体效能阻止犯罪 ${ }^{[33-36]}$ 。 加之,一个理性的街头抢劫者不太可能到一个犯罪 防控水平高的区域犯罪 ${ }^{[15,72]}$ 。因此,理性的街头抢 劫者可能会分析“前案件”区域的居民集体效能、警 察日常巡逻、公共安全视频监控, 以及逃离犯罪现 场的难易程度等, 从而促使其不再到当场被捕的 “前案件”区域实施犯罪。

\section{参考文献(References)}

[1] Townsley M, Birks D, Bernasco W, et al. Burglar target selection: A cross- national comparison [J]. Journal of Research in Crime and Delinquency, 2015, 52(1): 3-31.

[2] Bernasco W. A sentimental journey to crime: Effects of residential history on crime location choice [J]. Criminology, 2010, 48(2): 389-416.

[3] Bernasco W, Block R, Ruiter S. Go where the money is: Modeling street robbers' location choices [J]. Journal of Economic Geography, 2013, 13(1): 119-143.

[4] Deakin J, Smithson H, Spencer J, et al. Taxing on the streets: Understanding the methods and process of street robbery $[\mathrm{J}]$. Crime Prevention and Community Safety, 2007, 9(1): 52-67.

[5] 徐冲, 柳林, 周素红, 等. DP 半岛街头抢劫犯罪案件热点 时空模式 [J]. 地理学报, 2013, 68(12): 1714-1723. [Xu Chong, Liu Lin, Zhou Suhong, et al. The spatio-temporal patterns of street robbery in DP Peninsula. Acta Geographica Sinica, 2013, 68(12): 1714-1723. ]

[6] 徐冲, 柳林, 周素红, 等. 微观空间因素对街头抢劫影响 的空间异质性: 以 DP 半岛为例 [J]. 地理研究, 2017, 36 (12): 2492-2504. [Xu Chong, Liu Lin, Zhou Suhong, et al. Spatial heterogeneity of micro-spatial factors' effects on street robberies: A case study of DP Peninsula. Geo- 
graphical Research, 2017, 36(12): 2492-2504. ]

[7] Haberman C P, Ratcliffe J H. Testing for temporally differentiated relationships among potentially criminogenic places and census block street robbery counts $[\mathrm{J}]$. Criminology, 2015, 53(3): 457-483.

[8] Bernasco W, Ruiter S, Block R. Do street robbery location choices vary over time of day or day of week? A test in Chicago [J]. Journal of Research in Crime and Delinquency, 2017, 54(2): 244-275.

[9] 龙冬平. 街头抢劫者作案地选择的时空特征与形成机理 [D]. 广州: 中山大学, 2018. [Long Dongping. Research on the spatio-temporal characteristic and formation mechanism of crime location choice of street robbers. Guangzhou, China: Sun Yat-Sen University, 2018. ]

[10] Lammers M, Menting B, Ruiter S, et al. Biting once, twice: The influence of prior on subsequent crime location choice [J]. Criminology, 2015, 53(3): 309-329.

[11] Bernasco W, Johnson S D, Ruiter S. Learning where to offend: Effects of past on future burglary locations [J]. Applied Geography, 2015, 60(5): 120-129.

[12] Bernasco W, Block R. Where offenders choose to attack: A discrete choice model of robberies in Chicago [J]. Criminology, 2009, 47(1): 93-130.

[13] Farrell G, Philips C, Pease K. Like taking candy: Why does repeat victimization occur? [J]. British Journal of Criminology, 1995, 35(3): 384-399.

[14] 龙冬平, 柳林, 周素红, 等. 地理学视角下犯罪者行为研 究进展 [J]. 地理科学进展, 2017, 36(7): 886-902. [Long Dongping, Liu Lin, Zhou Suhong, et al. Research progress of criminal behavior from the perspective of geography. Progress in Geography, 2017, 36(7): 886-902. ]

[15] Johnson D. The space/time behaviour of dwelling burglars: Finding near repeat patterns in serial offender data [J]. Applied Geography, 2013, 41(7): 139-146.

[16] Townsley M, Birks D, Ruiter S, et al. Target selection models with preference variation between offenders [J]. Journal of Quantitative Criminology, 2016, 32(2): 283-304.

[17] Block R, Block C R. The Bronx and Chicago: Street robbery in the environs of rapid transit stations [M]. Thousand Oaks, USA: Sage Publications, 1999.

[18] Kooi B R. Assessing the correlation between bus stop densities and residential crime typologies [J]. Crime Prevention and Community Safety, 2013, 15(2): 81-105.

[19] Groff E R, Ratcliffe J H, Haberman C P, et al. Does what police do at hot spots matter? The Philadelphia policing tactics experiment [J]. Criminology, 2015, 53(1): 23-53.

[20] Brantingham P L, Brantingham P J. Criminality of place: Crime generators and crime attractors [J]. European Journal on Criminal Policy and Research, 1995, 3(3): 5-26.

[21] Bernasco W, Block R. Robberies in Chicago: A block-lev- el analysis of the influence of crime generators, crime attractors, and offender anchor points [J]. Journal of Research in Crime and Delinquency, 2011, 48(1): 33-57.

[22] Kurland J, Johnson S D, Tilley N. Offenses around stadiums: A natural experiment on crime attraction and generation [J]. Journal of Research in Crime and Delinquency, 2014, 51(1): 5-28.

[23] Jean P K B S. Pockets of crime: Broken windows, collective efficacy, and the criminal point of view [M]. Chicago, USA: The University of Chicago Press, 2008.

[24] Gialopsos B M, Carter J W. Offender searches and crime events [J]. Journal of Contemporary Criminal Justice, 2015, 31(1): 53-70.

[25] Clarke R V, Cornish D B. Modeling offenders' decisions: A framework for research and policy [J]. Crime and Justice, 1985, 6: 147-185.

[26] Clarke R V, Felson M. Routine activity and rational choice: Advances in criminological theory (Vol. 5) [M]. New Brunswick, USA: Transaction Publisher, 1993.

[27] Felson M, Clarke R V. Opportunity makes the thief: Practical theory for crime prevention $[\mathrm{J}]$. Police Research, 1998, 1(1): 1-36.

[28] Morrison S, O'Donnell I. An analysis of the decisionmaking practices of armed robbers [M]. Monsey, USA: Criminal Justice Press, 1996.

[29] Johnson S D, Bowers K J. The stability of space- time clusters of burglary [J]. British Journal of Criminology, 2004, 44(1): 55-65.

[30] Jacobs B, Wright R. Stick-up, street culture, and offender motivation[J]. Criminology, 1999, 37(1): 149-174.

[31] Bursik R J. Social disorganization and theories of crime delinquency: Problems and prospects [J]. Criminology, 1988, 26(4): 519-552.

[32] Shaw C R, Mckay H D. Juvenile delinquency and urban areas [M]. Chicago, USA: University of Chicago Press, 1942.

[33] Hirschfield A, Bowers K J. The effect of social cohesion on levels of recorded crime in disadvantaged areas [J]. Urban Studies, 1997, 34(8): 1275-1295.

[34] Weisburd D, Groff E R, Yang S. Understanding and controlling hot spots of crime: The importance of formal and informal social controls [J]. Prevention Science, 2014, 15 (1): 31-43.

[35] Braga A A, Clarke R V. Explaining high-risk concentrations of crime in the city: Social disorganization, crime opportunities, and important next steps [J]. Journal of Research in Crime and Delinquency, 2014, 51(4): 480-498.

[36] Law J, Quick M, Chan P. Open area and road density as land use indicators of young offender residential locations at the small-area level: A case study in Ontario, 
Canada [J]. Urban Studies, 2016, 53(8): 1710-1726.

[37] Ceccato V, Haining R. Assessing the geography of vandalism: Evidence from a Swedish city [J]. Urban Studies, 2005, 42(9): 1637-1656.

[38] Galster G, Santiago A. Neighbourhood ethnic composition and outcomes for low-income Latino and African American children [J]. Urban Studies, 2017, 54(2): 482-500.

[39] Johnson S D, Summers L. Testing ecological theories of offender spatial decision making using a discrete choice model [J]. Crime \& Delinquency, 2015, 61(3): 454-480.

[40] Hirschfield A, Birkin M, Brunsdon C, et al. How places influence crime: The impact of surrounding areas on neighbourhood burglary rates in a British city [J]. Urban Studies, 2014, 51(5): 1057-1072.

[41] 柳林, 张春霞, 冯嘉欣, 等. ZG 市计骗犯罪的时空分布 与影响因素 [J]. 地理学报, 2017, 72(2): 315-328. [Liu Lin, Zhang Chunxia, Feng Jiaxin, et al. The spatial-temporal distribution and influencing factors of fraud crime in ZG City, China. Acta Geographica Sinica, 2017, 72 (2): 315-328. ]

[42] Bernasco W, Nieuwbeerta P. How do residential burglars select target areas? A new approach to the analysis of criminal location choice [J]. British Journal of Criminology, 2005, 45(3): 296-315.

[43] Johnson S D, Bernasco W, Bowers K J, et al. Space-time patterns of risk: A cross national assessment of residential burglary victimization $[\mathrm{J}]$. Journal of Quantitative Criminology, 2007, 23(3): 201-219.

[44] Johnson S D. Repeat burglary victimisation: A tale of two theories [J]. Journal of Experimental Criminology, 2008, 4(3): 215-240.

[45] Byeon J, Kim I, Lee D. Protest and property crime: Political use of police resources and the deterrence of crime [J]. Public Choice, 2018, 175(1-2): 181-196.

[46] Benson B L. The allocation of police [M]// Benson B L, Zimmerman P R. Handbook on the economics of crime. Cheltenham, UK: Edward Elgar, 2010: 184-217.

[47] Bernasco W. Them again? Same-offender involvement in repeat and near repeat burglaries [J]. European Journal of Criminology, 2008, 5(4): 411-431.

[48] Townsley M, Homel R, Chaseling J. Infectious burglaries: A test of the near repeat hypothesis [J]. British Journal of Criminology, 2003, 43(3): 615-633.

[49] Smith W, Bond J W, Townsley M. Determining how journeys- to- crime vary: Measuring inter-and intra- offender crime trip distributions [M]. New York, USA: Springer, 2009: 217-236.

[50] Ackerman J M, Rossmo D K. How far to travel? A multilevel analysis of the residence-to-crime distance [J]. Journal of Quantitative Criminology, 2015, 31(2): 237-262.
[51] Snook B, Wright M, House J C, et al. Searching for a needle in a needle stack: Combining criminal careers and journey to crime research for criminal suspect prioritization [J]. Police Practice and Research, 2006, 7(3): 217230 .

[52] Vandeviver C, Van Daele S, Vander Beken T. What makes long crime trips worth undertaking? Balancing costs and Benefits in burglars' journey to crime [J]. British Journal of Criminology, 2015, 55(2): 399-420.

[53] Wiles P, Costello A. The "road to nowhere": The evidence for travelling [M]. London, UK: Home Office, 2000.

[54] Van Koppen P J, Jansen R W J. The road to robbery: Travel patterns in commercial robberies [J]. British Journal of Criminology, 1998, 38(2): 230-246.

[55] Cornish D B, Clarke R V. The reasoning criminal: Rational choice perspectives on offending [M]. New York, USA: Springer, 1986.

[56] Liu L, Jiang C, Zhou S, et al. Impact of public bus system on spatial burglary patterns in a Chinese urban context [J]. Applied Geography, 2017, 89: 142-149.

[57] 龙冬平, 柳林, 冯嘉欣, 等. 社区环境对人室盗窃和室外 盗窃影响的对比分析: 以 ZG 市 ZH 半岛为例 [J]. 地理 学报, 2017, 72(2): 341-355. [Long Dongping, Liu Lin, Feng Jiaxin, et al. Comparisons of the community environment effects on burglary and outdoor- theft: A case study of ZH peninsula in ZG City. Acta Geographica Sinica, 2017, 72(2): 341-355. ]

[58] Baudains P, Braithwaite A, Johnson S D. Target choice during extreme events: A discrete spatial choice model of the 2011 London riots [J]. Criminology, 2013, 51(2): 251285.

[59] Groff E R, Lockwood B. Criminogenic facilities and crime across street segments in Philadelphia: Uncovering evidence about the spatial extent of facility influence [J]. Journal of Research in Crime and Delinquency, 2014, 51 (3): 277-314.

[60] Andresen M A, Malleson N. Police foot patrol and crime displacement [J]. Journal of Contemporary Criminal Justice, 2014, 30(2): 186-199.

[61] Braga A A. The continued importance of measuring potentially harmful impacts of crime prevention programs: the academy of experimental criminology 2014 Joan McCord lecture [J]. Journal of Experimental Criminology, 2016, 12(1): 1-20.

[62] Haberman C P, Groff E R, Ratcliffe J H, et al. Satisfaction with police in violent crime hot spots: Using community surveys as a guide for selecting hot spots policing tactics [J]. Crime \& Delinquency, 2016, 62(4): 525-557.

[63] Jacobs B A, Addington L A. Gating and residential robbery [J]. Crime Prevention \& Community, 2016, 18(1): 
19-37.

[64] 聂冲, 贾生华. 离散选择模型的基本原理及其发展演进 评介 [J]. 数量经济技术经济研究, 2005(11): 151-159.

[Nie Chong, Jia Shenghua. Research on the theoretical basis and evolution of discrete choice models. The Journal of Quantitative \& Technical Economics, 2005(11): 151-159. ]

[65] Mcfadden D, Train K. Mixed M N L models for discrete response [J]. Journal of Applied Econometrics, 2000, 15 (5): 447-470.

[66] 王灿, 王德, 朱玮, 等. 离散选择模型研究进展 [J]. 地理 科学进展, 2015, 34(10): 1275-1287. [Wang Can, Wang De, Zhu Wei, et al. Research progress of discrete choice models. Progress in Geography, 2015, 34(10): 12751287. ]

[67] Frith M J, Johnson S D, Fry H M. Role of the street network in Burglars' spatial decision-making [J]. Criminolo- gy, 2017, 55(2): 344-376.

[68] McFadden D. Quantitative methods for analysing travel behaviour of individuals: Some recent developments [M]// Henser D A, Stopher P R B. Vioural travel modelling. London, UK: Croom Helm, 1978: 279-318.

[69] Hamilton L C. Statistics with STATA: Version 12 [M]. California, USA: Cengage Learning, 2012.

[70] Van Daele S, Beken T V. Outbound offending: The journey to crime and crime sprees [J]. Journal of Environmental Psychology, 2011, 31(1): 70-78.

[71] Liu L, Li J C M. Progress and future directions of crime research in China with selected case studies [J]. Journal of Research in Crime and Delinquency, 2017, 54(4): 447453.

[72] Johnson S D, Summers L, Pease K. Offender as forager? A direct test of the boost account of victimization [J]. Journal of Quantitative Criminology, 2009, 25(2): 181-200.

\title{
Impact of prior crime experiences of street robbers on subsequent crime location choices
}

\author{
LONG Dongping ${ }^{1}$, LIU Lin ${ }^{1,2^{*}}$, CHEN Jianguo ${ }^{1}$, XIAO Luzi ${ }^{1}$, SONG Guangwen ${ }^{1}$, XU Chong ${ }^{1}$ \\ (1. School of Geographical Science, Center of GeoInformatics for Public Security, Guangzhou University, Guangzhou 510275, China; \\ 2. Department of Geography, University of Cincinnati, Cincinnati OH 45221-0131, Ohio, USA)
}

\begin{abstract}
Explaining the choice of crime location is one of the central themes of crime geography. The existing research on the choice of the location where street robbers commit crimes mainly focuses on the following two aspects: analyzing the spatial pattern and influencing factors from a comprehensive perspective, and analyzing the time difference from a comparative perspective. In general, these studies not only enrich the research perspectives, but also clarify the spatiotemporal patterns and their formation mechanisms. Therefore, they have important theoretical and practical significance. The literature also shows that offenders' subsequent crime location choices are affected by their prior crime location choices. However, the published studies have focused on the influences of time and place of a previous crime, and have not yet verified the role of crime experiences of the former offense. Therefore, this study further examined the influence of the prior individual robbery experiences on the subsequent street robbery location choices by using a mixed logit model and data on arrested robbers in ZG City, China. The results suggest that the individual criminal experiences of street robbers such as the interval of crimes, criminal travel, and arrest on the spot have a strong effect on subsequent street robbery location choices, that is, a shorter time interval, a shorter distance of journey to prior crime location, and less possibility of being arrested in the act of a prior street robbery significantly increase the likelihood of a robber returning to the previous location. Finally, through police interviews and theoretical analysis, it is found that the last situation is formed by the offenders' fear of being arrested on the spot, the special ways of police intervention, as well as the social cohesion and crime prevention of communities. Therefore, the extension of the results may well provide references for the police departments' work on prevention and pre-event control and active intervention.
\end{abstract}

Keywords: street robbers; crime experiences; crime location choices; mixed logit model; crime geography 